\title{
EXTRACTION OF POLYCYCLIC AROMATIC HYDROCARBONS FROM WATER SAMPLES INTO A ROTATING-DISK MICROEXTRACTOR AND THE SUBSEQUENT DETERMINATION BY GAS CHROMATOGRAPHY-MASS SPECTROMETRY
}

\author{
YANINA CORROTEA ${ }^{1}$, KAREN SÁNCHEZ $^{2}$, M. ANGÉLICA RUBIO ${ }^{2}$, PABLO RICHTER ${ }^{1, *}$ \\ ${ }^{1}$ Departamento de Química Inorgánica y Analítica, Facultad de Ciencias Químicas y Farmacéuticas, Universidad de Chile, Casilla 233, Santiago, Chile. \\ ${ }^{2}$ Departamento Ciencias del Ambiente, Facultad de Química y Biología, Universidad de Santiago de Chile, Santiago, Chile.
}

\begin{abstract}
The rotating disk sorptive extraction (RDSE) technique was applied in this study to the extraction of 16 polycyclic aromatic hydrocarbons (PAHs) in real water samples. The sorptive and desorptive behavior of the analytes were studied by using a rotating disk coated with polydimethylsiloxane (PDMS) on one of its surfaces. The optimal conditions for extractions of all analytes were: rotational velocity of the disk: $1250 \mathrm{rpm}$; sample volume: $20 \mathrm{~mL}$; matrix modifier: 20 $\% \mathrm{MeOH}$ and extraction time: $30 \mathrm{~min}$. A desorption time of $10 \mathrm{~min}$ at $1250 \mathrm{rpm}$ was used for the analytes using $5 \mathrm{~mL}$ of methanol. PAHs were measured in the extract by gas chromatography coupled to mass spectrometry (GC-MS). Under these conditions, recoveries ranging from 84 to $100 \%$ were obtained in drinking water enriched with the analytes, and the precision, expressed as relative standard deviation, was between 2 and $20 \%$. The detection limit of the method was between 8 and $115 \mathrm{ng} \mathrm{L}^{-1}$ depending on the analyte. The method was applied to the analysis of water samples obtained from a lagoon in Curauma, Chile, obtaining quantitative recoveries.
\end{abstract}

Keywords Rotating disk sorptive extraction, Polydimethylsiloxane, PAHs, Water samples.

\section{INTRODUCTION}

PAHs are a group of chemical compounds composed of carbon and hydrogen, which form aromatic cyclic structures with two or more fused rings and can exist in several isomeric arrangements ${ }^{1}$. They can be found in oil, coal, tar deposits as well as products of fuel utilization. Incomplete combustion of organic matter at high temperature is one of the major anthropogenic sources of environmental $\mathrm{PAHs}^{2}$. Exposures to PAHs have been identified to associate with increased cancer risk of the skin, bladder and lung ${ }^{3}$. Only 16 of them have been selected as priority contaminants by the US Environmental Protection Agency (EPA) and by the European Union (EU) on the basis of their occurrence and carcinogenicity ${ }^{4}$, which are associated with vehicular pollution and cigarette smoke $\mathrm{s}^{5}$ Their molecular weight and lipophilic character are the most important factors regarding toxicity ${ }^{6}$. Some of PAHs can cause cancers in humans and are harmful to fish and other aquatic life ${ }^{7}$.

PAHs are released into the atmosphere as airborne particles and gases. Some of them are ultimately removed from the air through dry and wet deposition to other compartments of the environment. Therefore, monitoring the levels of PAHs contents in water system are required to ensure human health and environmental control. In this context, to guarantee the quality of drinking water, EU Directive proposes, inter alia, the evaluation of five PAHs establishing the maximum concentration limits ${ }^{8}$.

Different modern analytical methods have been described for the extraction of PAHs in liquid samples, among them solid-phase microextraction $(\mathrm{SPME})^{6,9,10}$, solid-phase extraction (SPE) ${ }^{11}$ and stir-bar-sorptive extraction $(\mathrm{SBSE})^{3,4,7,12}$ have been described. These modern techniques have the advantage over the conventional extraction methods in terms of solvent usage and removal time. In our laboratory we have recently developed the technology of rotating disk sorptive extraction (RDSE), an alternative microextraction technique similar to SPME and $\mathrm{SBSE}^{13}$. The rotating disk extractor has the advantage of providing a greater volume of polydimethylsiloxane (PDMS) than in SPME, while also having a larger PDMS surface area than that provided by the device used in $\mathrm{SBSE}^{13-18}$. On the other hand, the disk can be rotated at high velocity without risk of damaging the PDMS phase, because it is only in contact with the solution, which facilitates more efficient analyte mass transfer and faster extraction $^{19}$. In the case of the device used in SBSE, the friction of the phase with the bottom of the vessel containing the sample decreases its durability ${ }^{13}$. Also the RDSE can reduce solvent usage in sample preparation steps and the disk configuration is very easy to make in the laboratory ${ }^{13-18}$.

The aim of this study was to develop a method for PAHs determination from aqueous matrices. The analytes were extracted by RDSE, them desorbed from the PDMS with methanol and detected by gas chromatography coupled to mass spectrometry (GC-MS).

\section{MATERIALS AND METHODS}

\subsection{Reagents}

High-purity water from a Millipore Milli-Q PLUS ultrapure water system (Billerica, MA) was used throughout the work. The 16 PAHs examined in this study were: Naphthalene (Nap), acenaphthylene (Apy), acenaphtene (AcP), fluorene (Flu), phenanthrene (Phe), anthracene (Ant), fluoranthene (FL), pyrene (Pyr), benzo(a)anthracene (BaA), chrysene ( $\mathrm{Chr})$, benzo(b)fluoranthene $(\mathrm{BbF})$, benzo(k)fluoranthene $(\mathrm{BkF})$, benzo(a)pyrene $(\mathrm{BaP})$, indeno[1,2,3-cd]pyrene (InP), dibenz[a,h]anthracene (DBahA), benzo[g,h,i]perylene (BghiP) and were purchased from Dr Ehrenstorfer (Augsburg, Germany). A stock solution of $10 \mathrm{mg} \mathrm{L}^{-1}$ of the analytes was prepared in methanol. PCB 77 was used as internal standard at a concentration of $20 \mathrm{mg} \mathrm{L}^{-1}$ and was purchased from $\mathrm{Dr}$ Ehrenstorfer. The PAHs labeled mix, Acenaphtene-D10, Phenanthrene-D10, Crysene-D10, Perilene-D10, purchased from Dr Ehrenstorfer, was used as a surrogate standard for the analysis of real water samples at a concentration of $10 \mathrm{mg} \mathrm{L}^{-1}$ in methanol. Nitrogen 5.0 and helium 5.0 were purchased from Linde (Santiago, Chile) and were used for final extract evaporation and as chromatographic carrier gas, respectively. Methanol, acetone (both HPLCgrade, $99.8 \%$ purity) and sodium chloride (99.5\% purity) were purchased from Merck (Darmstadt, Germany). The PDMS phase was prepared from a Sylgard 184 silicone elastomer kit (Dow Corning, MI, USA) according to the recommendations of the manufacturer.

\subsection{Instrumental and software}

A Thermo Scientific gas chromatograph model Focus (Milan, Italy) coupled to a Thermo Fisher Scientific model ISQ (Austin, TX, USA) massselective detector was used for final determinations. The fused silica capillary column used was a Restek (Bellefonte, PA, USA) RTX-5MS (30m x $0.25 \mathrm{~mm}$ id.; $0.25 \mu \mathrm{m}$ film thickness) coated with $5 \%$ phenyl-95 methylpolysiloxane. Two microliters of samples extract was injected into the gas chromatograph using the splitless mode. The injector temperature was $250{ }^{\circ} \mathrm{C}$. The initial column temperature was $70{ }^{\circ} \mathrm{C}$, at a rate of $10{ }^{\circ} \mathrm{C} \min ^{-1}$ to $260{ }^{\circ} \mathrm{C}$, it was increased to $300{ }^{\circ} \mathrm{C}$ at a rate of $5{ }^{\circ} \mathrm{C} \mathrm{min}{ }^{-1}$ and held for $8 \mathrm{~min}$. A constant flow of $1.0 \mathrm{~mL} \mathrm{~min}{ }^{-1}$ helium was used as carrier gas. The solvent delay was 7 min. A dwell time of $0.1 \mathrm{~s}$ was used for each $\mathrm{m} / \mathrm{z}$ value. The MS transfer line was maintained at $250{ }^{\circ} \mathrm{C}$ and quantification was based on calibration with the standard analyte using the mass spectrometer in selective ion monitoring (SIM) mode. Table 1 shows the ions monitored for the analytes and internal and surrogate standards.

2.3 Preparation of the rotating disk devices.

The extraction device used in this study was a Teflon disk $(1.5 \mathrm{~cm}$ diameter $)$ into which a miniature magnetic stirring bar (Teflon-coated Micro Stit bar from VWR International) was embedded. A film of PDMS was subsequently attached to one side of the disk with double-sided adhesive tape (Figure 1). 


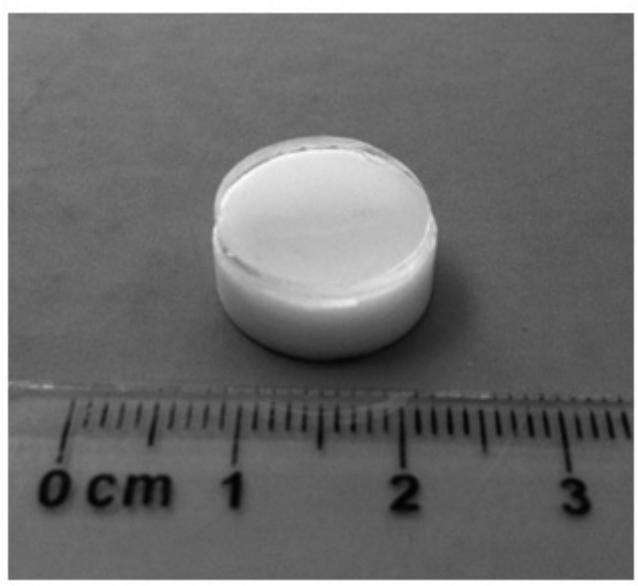

Figure 1. Photography of Rotating disk extractor made of Teflon containing PDMS on the surface and a miniature magnetic stirring bar inserted.

The PDMS films were prepared as follows: the ratio of base-to-catalyst mixture was 10:1 (w/w), and the curing time at room temperature was $48 \mathrm{~h}$ Before curing, the gel solution was poured into a square tile for PDMS gelation, in which the area is delimited by a rubber band with a width of $2 \mathrm{~mm}$. The thickness of the formed PDMS film may be modified by rubber band width. One circular part of the phase, equivalent to the desired area $(1.5 \mathrm{~cm})$, was cut using a hollow punch and fixed onto the Teflon disk using double-sided tape.

Table 1. GC-MS data for determination of PAHs.

\begin{tabular}{|c|c|c|c|}
\hline Analyte & Abbreviation & $\begin{array}{c}\text { Retention } \\
\text { time (min) }\end{array}$ & $\begin{array}{c}\text { Target and } \\
\text { qualifier ion } \\
m / z\end{array}$ \\
\hline Naphthalene & Nap & 6.9 & 128,129 \\
\hline Acenaphthylene & Apy & 10.52 & 152,153 \\
\hline Acenaphthene & Acp & 10.97 & 153,154 \\
\hline Fluorene & Flu & 12.16 & 166,165 \\
\hline Phenanthrene & Phe & 14.45 & 176,179 \\
\hline Anthracene & Ant & 14.56 & 176,179 \\
\hline Fluoranthene & FL & 17.36 & 202,203 \\
\hline Pyrene & Pyr & 17.89 & 202,203 \\
\hline Benz(a)anthracene & $\mathrm{BaA}$ & 20.97 & 228,226 \\
\hline Chrysene & Chr & 21.08 & 228,226 \\
\hline Benzo(b)fluoranthene & $\mathrm{BbF}$ & 24.19 & 252,253 \\
\hline Benzo(k)fluoranthene & $\mathrm{BkF}$ & 24.26 & 252,253 \\
\hline Benzo(a)pyrene & $\mathrm{BaP}$ & 25.17 & 252,253 \\
\hline indeno(1 23 -cd)pyrene & $\mathrm{InP}$ & 28.78 & 276,277 \\
\hline Dibenzo(a,h)anthracene & DBah & 28.88 & 278,277 \\
\hline Benzo(g,h,i)perylene & BghiP & 29.72 & 276,277 \\
\hline Acenaphthene- $\mathrm{D}_{10}$ & & 10.89 & 164,162 \\
\hline Phenanthrene- $\mathrm{D}_{10}$ & & 14.40 & 188,189 \\
\hline Chrysene- $\mathrm{D}_{12}$ & & 21.01 & 240,236 \\
\hline Perylene- $\mathrm{D}_{12}$ & & 25.35 & 264,260 \\
\hline $\mathrm{PCB}_{77}$ & & 18.52 & 290,292 \\
\hline
\end{tabular}

2.4 General procedure

A standard or water sample $(20 \mathrm{~mL})$ was poured into a beaker containing $5 \mathrm{~mL}$ of methanol. Then an aliquot of surrogate standard solution was added to reach a concentration of $2 \mu \mathrm{g} \mathrm{L}^{-1}$ in the sample. The rotating disk coated with the PDMS phase was placed inside the beaker, and the disk was rotated at $1,250 \mathrm{rpm}$ for $30 \mathrm{~min}$ at room temperature. After extraction, the disk was placed in a $10 \mathrm{~mL}$ beaker containing $5 \mathrm{~mL}$ methanol as a desorbing solvent, and was stirred for $10 \mathrm{~min}$ at $1,250 \mathrm{rpm}$. Desorption was repeated with another 5 $\mathrm{mL}$ methanol. Both methanol extracts containing the concentrated analyte were then joined and evaporated to $0.5 \mathrm{~mL}$ under a stream of $\mathrm{N}_{2}$. Before injection the internal standard PCB 77 was added, and the analytes were determined by GC-MS.

In the study of the variables the concentration of the analytes was $5 \mu \mathrm{g} \mathrm{L}^{-1}$. 2. 5 Determination of PAHs in a real sample

The method was applied to the determination of PAHs in a surface water sample. The sample was obtained from the lagoon La Luz, in Curauma (Valparaíso, Chile). The lagoon is located in the $\mathrm{V}$ region of Valparaiso, Chile. The water sample was stored in $3 \mathrm{~L}$ ambar glass bottles and carried to the laboratory in an ice box. It was stored at $4^{\circ} \mathrm{C}$ until analysis. Prior to extraction, the samples were filtered through a Whatman GF/C filter using a Buchner flask. The sample was subjected to the extraction/desorption procedure using the optimum conditions as described previously. Subsequently, another portion of this sample was enriched with analytes at a concentration of $5 \mu \mathrm{g} \mathrm{L}^{-1}$ to determine the recovery of each analyte in a real sample matrix.

\section{RESULTS AND DISCUSSION}

3.1 Influence of the addition of $\mathrm{NaCl}$ and methanol.

PDMS polymeric phase preferably allows extraction of non-polar analytes. The matrix modifier $\mathrm{NaCl}$ is used to change the ionic strength of the sample, allowing that semi-polar and polar analytes increases their extraction efficiency into PDMS, by making the analytes insoluble in water and increasing their affinity for the organic phase. Because all the PAHs under study are hydrophobic ( $\log \mathrm{Kow}>4$ ), it was confirmed that presence of $\mathrm{NaCl}$ reduced the analyte extraction. Similar results were obtained by other authors using PDMS in both $\mathrm{SBSE}^{4,12}$ and $\mathrm{SPME}^{9}$, where the addition of $\mathrm{NaCl}$ decreased the recovery of the PAHs studied. Consequently further experiments were performed in absence of salt.

Addition of $\mathrm{MeOH}$ was also evaluated as matrix modifier because this solvent prevents adsorption of apolar analytes on the glass wall ${ }^{20,21}$. Three levels of methanol, $10 \%, 20 \%$ and $25 \%$ were analyzed; finding that the higher recoveries for the studied analytes were obtained with $20 \% \mathrm{MeOH}$. Higher concentrations of methanol decrease the extraction efficiency in PDMS.

3.2 Influence of the rotational velocity of the disk on analyte response

It is important to consider the rotational velocity of the disk, because indicates the efficiency of the mass transfer of the analyte from the aqueous phase to the polymeric phase. Figure 2 shows the effect of the rotational velocity of the disk on the extraction of PAHs. In this case, some representative PAHs were selected having low, medium and high molecular weight. The study was performed up to $3000 \mathrm{rpm}$, taking into account that the disk can be stirred at higher velocities without damaging the phase ${ }^{13-18}$. It can be observed that by increasing the rotational velocity the extraction efficiency of PAHs increased to reach a constant value at approximately $1250 \mathrm{rpm}$. 


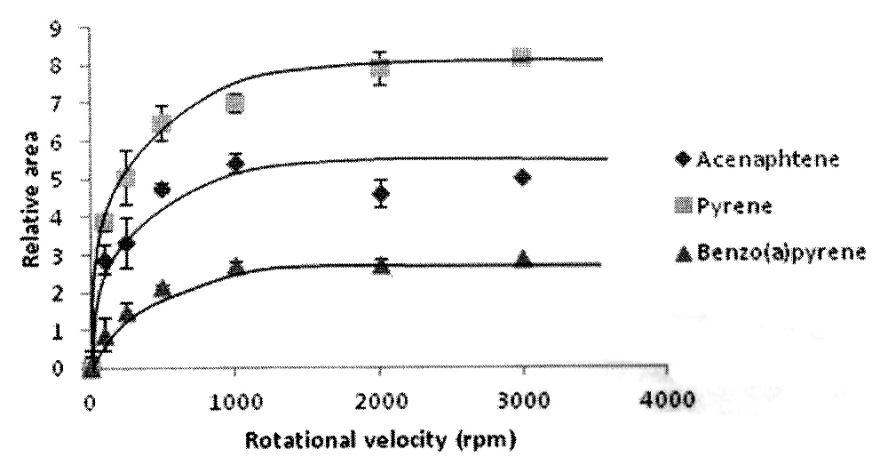

Figure 2. Effect of the rotational velocity of the disk on the analyte response. Error bars are given as standard deviation (3 replicates).

\subsection{Influence of extraction time}

The time at which the partition equilibrium of the extraction is reached was studied between 15 and 60 min. Regarding this variable, an extraction time of 30 min was sufficient to reach the partition equilibrium for sample volumes of $20 \mathrm{~mL}$. Compared with the extraction of more polar analytes ${ }^{13-18}$, the equilibrium time for PAHs is reached faster, because these analytes have an enhanced affinity with PDMS, facilitating their extraction. This equilibrium time was also significantly lower than in previous studies using $\mathrm{SBSE}^{3,4}$ because the surface area to phase volume ratio and the stagnant layer surface are improved under the RDSE configuration.

\subsection{Analytical figures of merit}

The analytical figures of merit of the proposed method are shown in Table 2. The calibration curve was constructed for each PAH at a concentration range ranging from 0.125 to $25 \mu \mathrm{g} \mathrm{L}^{-1}$, in water phase. The high values of the correlation coefficients indicate that the developed method is linear in the studied interval of concentrations. The accuracy and precision of method were determined in drinking water samples of Santiago city, which were spiked at $5 \mu \mathrm{g} \mathrm{L}{ }^{-1}$. A good accuracy was obtained at the spiked concentration, with values ranging from 84 to $100 \%$. Regarding to the precision of the method, it was determined as the repeatability of results from the recovery experiments, and was expressed as relative standard deviation (RSD, \%). The RSDs were between 2 and $20 \%$ where the less precise values were obtained for the three more hydrophobic analytes, similarly to the observed in other previous studies $^{12}$.

Table 2. Analytical features for the determination of PAHs

\begin{tabular}{|c|c|c|c|c|c|}
\hline PAH & $\begin{array}{c}\text { Linearity } \\
\left(\mathbf{R}^{\mathbf{2}}\right)\end{array}$ & $\begin{array}{c}\text { \%RSD } \\
(\mathbf{n}=\mathbf{3})\end{array}$ & $\begin{array}{c}\text { Recovery } \\
(\mathbf{\%}) \mathbf{( n = 3 )}\end{array}$ & $\begin{array}{c}\text { LOD } \\
\left(\mathbf{n g} \mathbf{L}^{-1}\right)\end{array}$ & $\begin{array}{c}\text { LOQ (ng } \\
\left.\mathbf{L}^{-1}\right)\end{array}$ \\
\hline Nap & 0.9997 & 6 & 94 & 8.0 & 10.6 \\
\hline Apy & 0.9984 & 7 & 88 & 16.4 & 47.4 \\
\hline AcP & 0.9989 & 2 & 96 & 21.8 & 59.5 \\
\hline Flu & 0.9991 & 2 & 100 & 75.7 & 238.2 \\
\hline Phe & 0.9978 & 6 & 96 & 36.4 & 76.7 \\
\hline Ant & 0.9961 & 9 & 92 & 79.0 & 235.2 \\
\hline FL & 0.9961 & 2 & 98 & 34.9 & 39.0 \\
\hline Pyr & 0.996 & 2 & 98 & 28.4 & 59.8 \\
\hline BaA & 0.9943 & 10 & 98 & 41.9 & 61.6 \\
\hline Chr & 0.9957 & 9 & 84 & 42.6 & 47.7 \\
\hline B(b)F & 0.9912 & 8 & 100 & 85.3 & 205.6 \\
\hline BkF & 0.9907 & 7 & 86 & 115.8 & 184.7 \\
\hline BaP & 0.9884 & 2 & 100 & 108.4 & 282.9 \\
\hline InP & 0.9943 & 20 & 100 & 54.2 & 96.9 \\
\hline DBahA & 0.9934 & 20 & 100 & 98.2 & 145.4 \\
\hline Bg.h.iP & 0.9935 & 20 & 100 & 61.9 & 88.3 \\
\hline
\end{tabular}

Detection (LOD) and quantification (LOQ) limits of the method (for 20 $\mathrm{mL}$ sample volume) were determined according to the IUPAC criterion ( $3 \sigma$ and $10 \sigma$, respectively). The detection limits obtained were between 8 and 115 ng L ${ }^{-1}$, depending on the analyte. To reach lower detection limits the sample volume should be increased.

3.5 Determination of PAHs in real samples

The extraction method was applied to determine PAHs in water samples from a lagoon. The result can be observed in Table 3 .

Only three PAHs were detected, other PAHs were under the LOD of the method. The recoveries of the different analytes, in the real water samples enriched with $5 \mu \mathrm{g} \mathrm{L}^{-1}$ concentration of PAHs, are in accordance with those obtained during optimization of the method.

Table 3. Concentration and recovery of PAHs in real lagoon water samples. Recovery were calculated after adding a concentration of $5 \mu \mathrm{g} \mathrm{L}^{-1}$ of each analyte. (Mean of 3 replicates).

\begin{tabular}{|c|c|c|}
\hline Compound & $\begin{array}{c}\text { Concentration } \\
\left(\mathbf{n g} \mathbf{L}^{-1} \pm \mathbf{S D}\right)\end{array}$ & Recovery (\% $\mathbf{\text { SD}})$ \\
\hline $\mathrm{Nap}$ & $240 \pm 20$ & $78 \pm 5$ \\
\hline $\mathrm{Apy}$ & $\mathrm{Nd}$ & $74 \pm 5$ \\
\hline $\mathrm{AcP}$ & $640 \pm 40$ & $92 \pm 6$ \\
\hline Flu & $\mathrm{Nd}$ & $100 \pm 6$ \\
\hline Phe & $1300 \pm 100$ & $92 \pm 2$ \\
\hline Ant & $\mathrm{Nd}$ & $78 \pm 2$ \\
\hline $\mathrm{FL}$ & $\mathrm{Nd}$ & $94 \pm 2$ \\
\hline $\mathrm{Pyr}$ & $\mathrm{Nd}$ & $96 \pm 2$ \\
\hline $\mathrm{BaA}$ & $\mathrm{Nd}$ & $92 \pm 2$ \\
\hline $\mathrm{Chr}$ & $\mathrm{Nd}$ & $78 \pm 2$ \\
\hline $\mathrm{B}(\mathrm{b}) \mathrm{F}$ & $\mathrm{Nd}$ & $100 \pm 8$ \\
\hline $\mathrm{BkF}$ & $\mathrm{Nd}$ & $87 \pm 1$ \\
\hline $\mathrm{BaP}$ & $\mathrm{Nd}$ & $100 \pm 8$ \\
\hline $\mathrm{InP}$ & $\mathrm{Nd}$ & $90 \pm 17$ \\
\hline $\mathrm{DBahA}$ & $\mathrm{Nd}$ & $98 \pm 10$ \\
\hline $\mathrm{Bg}, \mathrm{h}, \mathrm{iP}$ & $\mathrm{Nd}$ & $88 \pm 9$ \\
\hline & & \\
\hline
\end{tabular}

SD: Standard deviation

A critical comparison between the proposed method with other previously reported involving modern extraction devices such as the used in SBSE and SPME was performed indicating that the present method is an actual alternative to extract PAHs from water samples (Table 4). Recoveries in RDSE were one of the higher compared with SBSE and SPME, because the greater incorporation of PDMS to the disk with respect to the other devices. The LODs of the present method are comparable with other microextraction techniques, except when thermal desorption was used instead solvent desorption. Regarding rapidity in sample preparation, the present method is one of the faster methodologies, which is comparable with SPME when this technique was coupled to microwave assisted heating ${ }^{24}$ or conventional heating ${ }^{9}$. Finally RDSE is more economical, considering the disk configuration if easily fabricated in the laboratory.

\section{CONCLUSIONS}

The application of the technique RDSE for the determination of PAHs in water samples was established. The quite good analytical features achieved indicate that the method is an actual alternative to other modern extraction technology to determine PAHs from water samples.

Finally, this extraction technique has the advantage of being more economical because it can easily be synthesized at laboratory and the phase can be easily changed after each experiment from the disk surface. 
Table 4. Comparison between RDSE and other extraction techniques for PAHs from water.

\begin{tabular}{|c|c|c|c|c|c|c|c|}
\hline SBSE & 8 & $120 \mathrm{~min}$ & $0.5-7.3$ & $<9$ & $43-57$ & Drinking & [7] \\
\hline SBSE & 16 & $12 \mathrm{~h}$ & $0.4-14.7$ & $3-19$ & $97-122$ & Ultrapure & {$[20]$} \\
\hline SBSE & 6 & $14 \mathrm{~h}$ & $0.6-1,5$ & $5-23$ & NR & Surface & {$[22]$} \\
\hline SPME & 16 & $60 \mathrm{~min}$ & $1-41$ & $5-15.6$ & $78-109$ & Ultrapure & {$[24]$} \\
\hline SPME & 16 & $30 \mathrm{~min}$ & $30-1000$ & $5-13$ & $88-103$ & Wastewater & [25] \\
\hline RDSE & 16 & $30 \mathrm{~min}$ & $8-115$ & $2-20$ & $84-100$ & Drinking & This study \\
\hline
\end{tabular}

NR, not reported

\section{ACKNOWLEDGEMENTS}

The authors thank FONDECYT (project 1040716) for financial support.

\section{REFERENCES}

1. R. Simon, J. A. Gómez-Ruiz, T. Wenzl, Food Chem. 123, 819, (2010).

2. R. Ballesteros, J. J. Hernández, L. L. Lyons, Atmos. Environ. 43, 655, (2009).

3. G. Zhao, Y. Chen, S. Wang, J. Yu, X. Wang, F. Xie, H. Liu, J. Xie, Talanta. 116, 822, (2013).

4. Z. V. Gomes, L. Montero, C. Bauer, P. Poop, J. Chromatogr. A. 1091, 2, (2005).

5. K. Ravindra, R. Sokhi., R. Van Grieken, Atmos. Environ. 42, 2895, (2008).

6. P. Viñas, N. Campillo, N. Aguinaga, E. Pérez-Cánovas, M. HenándezCórdoba, J. Chromatogr. A. 1164, 10 (2007).

7. M .S. García-Falcón, B. Cancho-Grande, J. Simal-Gándara, Wat. Res. 38, 1679, (2004).

8. Official Journal of the European Communities (OJEC). Council Directive 98/83EC ( $3^{\text {rd }}$ November, 1998 ) relative to the quality of water intended for human consumption, L-330, $5^{\text {th }}$ December, 1998.

9. X. Cheng, J. Forsythe, E. Peterkin, Wat. Res. 47, 2331, (2013).

10. A. Ishizaki, K. Saito, N. Hanioka, S. Narimatsu, H. Kataoka, J. Chromatogr. A. 1217, 5555, (2010).

11. J. Ma, R. Xiao, J. Li, J. Yu, Y. Zhang, J. Chromatogr. A. 1217, 5462, (2010).
12. N. Barco-Bonilla, R. Romero-Gónzalez, P. Plaza-Bolaños, J. L. FernándezMoreno, A. Garrido, J. Martínez, Anal. Chim. Acta. 693, 62, (2011).

13. P. Richter, C. Leiva, C. Choque, A. Giordano, B. Sepúlveda, J. Chromatogr. A. 1216, 8598, (2009).

14. P. Richter, A. Cañas, C. Muñoz, C. Leiva, I. Ahumada, Anal. Chim. Acta. 695, 73, (2011).

15. A. Giordano, P. Richter, I. Ahumada, Talanta. 85, 2425, (2011).

16. A. Cañas, P. Richter, Anal. Chim. Acta. 743, 75, (2012).

17. V. Manzo, O. Navarro, L. Honda, K. Sánchez, M. I. Toral, Talanta. 106 , 305, (2013).

18. L. Jachero, B. Sepúlveda, I. Ahumada, E. Fuentes, P. Richter, Anal. Bioanal. Chem. 405, 7711, (2013).

19. L. Jachero, I. Ahumada, P. Richter, Anal. Bioanal. Chem. 406, 2987, (2014).

20. A. Prieto, O. Basauri O., Rodil R., A. Usobiaga, L. A. Fernández, N. Etxebarria, O. Zuloaga, J. Chromatogr. A. 1217, 2642, (2010).

21. P. Serodio, J. M. F. Nogueira, Anal. Chim. Acta. 517, 21, (2004).

22. V. M. León, J. Llorca-Pórcel, B. Álvarez, M. A. Cobollo, S. Muñoz, Anal. Chim. Acta. 558, 261, (2006).

23. A. J. King, J. W. Readman, J. L. Zhou, Anal. Chim. Acta. 523, 259, (2004).

24. E. Rianawati, R. Balasubramanian, Phys. Chem. Earth. 34, 857, (2009).

25. W. Ming-Chi, J. Jen-Fon, Talanta. 72, 1269, (2007). 